\title{
Quality of Life and Quality of Recovery in
}

\section{patients submitted to curative neoplastic surgery}

Azevedo J.', Sousa M.', Silva D.', Braga A.', Santos A.', Abelha F. 1,2

ICentro Hospitalar de São João, Dept of Anaesthesiology, Porto, Portugal

2Faculdade de Medicina da Universidade do Porto, Portugal

\section{Background and Goal of Study}

Quality of recovery is an important measure of the early postoperative health status. Our aim was to determine the impact of preoperative quality of life $(\mathrm{QoL})$ on quality of recovery on patients submitted to curative neoplastic surgery.

\section{Materials and Methods}

Prospective and observational study;

> Inclusion criteria: adult patients submitted to elective curative neoplastic surgery and admitted to the Post Anaesthetic Care Unit (PACU);

> Exclusion criteria: inability to give informed consent;

$>$ Quality of life before surgery:

EuroQol Five Dimensions Questionnaire (EQ-5D);

Poor quality of life (PQL) was defined as having problems in any of the 5 dimensions of EQ-5D.

> Frail patients (FP): Clinical Frailty Scale score $\geq 4$;

> Preoperative disability:

WHODAS SCORE $2.0 \geq 25 \%$.

> Postoperative recovery:

Postoperative Quality of Recovery Scale (PQRS)

at baseline (D0) and after surgery at minute 15 (TI5), 40 (T40) and days I (DI) and 3 (D3);

Recovery: return to baseline values for all questions in each domain;

Poor Quality of Recovery (PQR): absence of recovery to baseline values for at least three domains;

Quality of Recovery- I5 (QoR- I5) at baseline (D0) and after surgery at day I (DI).

> The Mann-Whitney $U$ test, Chi-square test or Fisher's exact test were used. Statistical significance was considered when $\mathrm{p}<0.05$.

\section{Results}

Of a total of I 48 patients, I 30 were considered with PQL (88\%).

\begin{tabular}{|c|c|c|c|}
\hline Variable & Non PQL N= 18 & $P Q L N=130$ & $P$ value \\
\hline $\begin{array}{l}\text { Age, median (years) } \\
{[\text { P25-P75] }}\end{array}$ & $\begin{array}{c}58,9 \\
{[49-70]}\end{array}$ & $\begin{array}{c}66 \\
{[55-76]}\end{array}$ & $0.076^{\mathrm{a}}$ \\
\hline $\begin{array}{l}\text { Gender male, N (\%) } \\
\text { Gender female, N (\%) }\end{array}$ & $\begin{array}{l}13(72) \\
5(27.8)\end{array}$ & $\begin{array}{l}52(40) \\
78(60)\end{array}$ & $0.0 I^{c}$ \\
\hline$A S A \geq 3, N(\%)$ & $3(16.7)$ & $48(36.9)$ & $0.090^{c}$ \\
\hline BMI, median [P25-P75] & $\begin{array}{c}25.95 \\
{[24,71-29.63]}\end{array}$ & $\begin{array}{c}25.71 \\
{[22.74-29.18]}\end{array}$ & $0.472^{a}$ \\
\hline $\begin{array}{c}\text { Disability (WHODAS 2.0) } \\
\mathrm{N}(\%)\end{array}$ & $0(0)$ & $32(24.46)$ & $0.013^{b}$ \\
\hline Frailty, N (\%) & $\mathrm{I}(5.5)$ & $44(33.8)$ & $0.013^{b}$ \\
\hline $\begin{array}{l}\text { EQVAS median } \\
\text { [P25-P75] }\end{array}$ & $80[65-90]$ & $80[50-76]$ & $0,002^{a}$ \\
\hline
\end{tabular}

Table 2 - Recovery according to Qor- 15 Univariate analysis

\begin{tabular}{|c|c|c|c|}
\hline QOR-I5 & $\begin{array}{c}\text { Non PQL } \\
\mathbf{N}=18\end{array}$ & $\begin{array}{c}\text { PQL } \\
N=130\end{array}$ & P Value \\
\hline $\begin{array}{l}\text { D0 total, median } \\
\text { [P25-P75] }\end{array}$ & $\begin{array}{c}142 \\
(138-147)\end{array}$ & $\begin{array}{c}127 \\
(1 \mid 2-136)\end{array}$ & $<0,\left.00\right|^{a}$ \\
\hline $\begin{array}{l}\text { DI total, median } \\
\text { [P25-P75] }\end{array}$ & $\begin{array}{c}118 \\
(113-134)\end{array}$ & $\begin{array}{c}113 \\
(95.5-126.5)\end{array}$ & $<0,08^{a}$ \\
\hline
\end{tabular}

Table 3 - Incomplete recovery according to PQRS Univariate analysis

\begin{tabular}{|c|c|c|c|c|}
\hline \multicolumn{2}{|c|}{$\begin{array}{l}\text { Incomplete Recovery } \\
\text { (domain/time frame) }\end{array}$} & \multirow{2}{*}{$\begin{array}{c}\text { Non PQL } \\
\begin{array}{c}\mathbf{N}=18 \\
\end{array} \\
77.78\end{array}$} & \multirow{2}{*}{$\begin{array}{c}\text { PQL } \mathbf{N}=130 \\
89.23\end{array}$} & \multirow{2}{*}{$\begin{array}{c}\text { P value } \\
0.238^{b}\end{array}$} \\
\hline \multirow{3}{*}{$\begin{array}{l}\text { Physiologic } \\
\text { (\%) }\end{array}$} & TI5 & & & \\
\hline & $\mathrm{T} 40$ & 66.67 & 73.44 & $0.546^{c}$ \\
\hline & DI & 23.53 & 26.98 & $0.510^{b}$ \\
\hline \multirow{4}{*}{$\begin{array}{c}\text { Nociceptive } \\
(\%)\end{array}$} & TI5 & II.II & 25.38 & $0.244^{b}$ \\
\hline & $\mathrm{T} 40$ & 33.33 & 28.13 & $0.648^{c}$ \\
\hline & DI & 17.65 & 27.78 & $0.560^{\mathrm{b}}$ \\
\hline & D3 & 27.78 & 25.41 & $0.780^{b}$ \\
\hline \multirow{4}{*}{$\begin{array}{c}\text { Emotional } \\
(\%)\end{array}$} & TI5 & 27.78 & 71.54 & $0.00 I^{b}$ \\
\hline & $\mathrm{T} 40$ & 22.22 & 72.66 & $<\left.0.00\right|^{b}$ \\
\hline & DI & 16.67 & 67.20 & $<\left.0.00\right|^{b}$ \\
\hline & D3 & II.II & 67.21 & $<\left.0.00\right|^{b}$ \\
\hline \multirow{4}{*}{$\begin{array}{c}\text { Cognitive } \\
(\%)\end{array}$} & TI5 & 94.44 & 92.31 & $0.603^{b}$ \\
\hline & $\mathrm{T} 40$ & 88.89 & 83.59 & $0.526^{b}$ \\
\hline & DI & 88.89 & 80.0 & $0.526^{b}$ \\
\hline & D3 & 88.24 & 78.05 & $0.525^{b}$ \\
\hline \multirow{3}{*}{$\begin{array}{l}\text { Functional } \\
\text { (\%) }\end{array}$} & $\mathrm{T} 40$ & 72.22 & 82.81 & $0.390^{\mathrm{b}}$ \\
\hline & DI & 55.56 & 68.8 & $0,263^{c}$ \\
\hline & D3 & 47.06 & 54.47 & $0.566^{c}$ \\
\hline
\end{tabular}

Table 4 - PQR at D3 according to PQRS

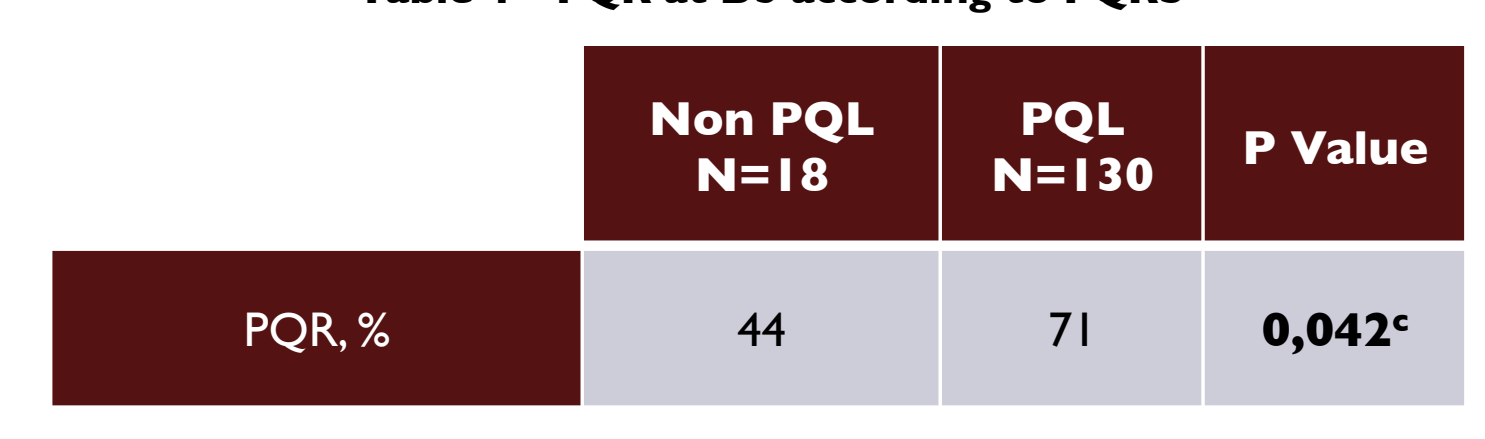

a: Mann-Whitney $U$ test test; b: Fisher's exact test; c: chi-squared test

\section{Conclusion}

Patients with poor quality of life presented more frailty, disability, and worst health status, before surgery.

According to PQRS they presented poor quality of recovery.

References:

I Rabin R., de Charro F., EQ-5D: a measure of health status from the EuroQol Group.AnnMed 200I; 33:337-43

II Royse, Collin F. et al, Development and Feasibility of a scale to Assess Postoperative Recovery. The Post-operative Quality Recovery Scale. Anesthesiology 2010 II3, 892-905 